\title{
Uma Análise dos Últimos 5 anos do Simpósio Brasileiro de Qualidade de Software
}

\author{
Maiane Salomão Carianha ${ }^{1}$, Crescencio Rodrigues Lima Neto ${ }^{1,2}$ \\ ${ }^{1}$ Instituto Federal da Bahia (IFBA) \\ Vitória da Conquista - Ba - Brasil \\ ${ }^{2}$ Departamento de Ciência da Computação - Universidade Federal da Bahia \\ (DCC/UFBA)
}

\begin{abstract}
In 2013, the Brazilian Symposium on Software Quality (SBQS, the acronym in Portuguese) performs its twelfth edition. This paper aims to analyze the technical papers and experience reports of the event last five editions. The method used was a systematic literature review published in the SQBS proceedings among 2008 and 2012. According to the research realized, we quantified the institutions from industry and academia that have published. We also observed the total number of publications, the state, the researcher and the institution that had published more. Finally, what were the languages used in the papers writing. Our analysis considered the electronic version of the SBQS last five editions and with the information gathered, we can draw the following conclusions: (i) the leading institutions are UFRJ, UFMG, and UFPE; (ii) the states that have more publications are Rio de Janeiro, Minas Gerais, and São Paulo; (iii) the relationship with the industry is still weak; (iv) the international participation is low; and finally, (v) There is a small amount of work written in English.
\end{abstract}

Resumo. No ano de 2013, o Simpósio Brasileiro de Qualidade de Software (SBQS) realiza sua décima segunda edição. Este artigo tem o objetivo de analisar os trabalhos técnicos e relatos de experiências das últimas cinco edições do evento. $O$ método utilizado foi a revisão sistemática da literatura publicada nos anais do SBQS entre 2008 e 2012. De acordo com a pesquisa realizada, quantificou-se as instituições que publicaram, destas, quais eram da indústria e quais eram unidades de ensino. Observou-se também o número total de publicações, o estado, o pesquisador e a instituição de ensino que mais publicou. Por fim, quais foram os idiomas utilizados na escrita dos artigos e relatos de experiência. Nossa análise considerou a versão eletrônica das últimas cinco edições do SBQS e com a informação levantada, pode-se tirar as seguintes conclusões: (i) as instituições que lideram são UFRJ, UFMG e UFPE; (ii) os estados que mais apresentam publicações são Rio de Janeiro, Minas Gerais e São Paulo; (iii) a relação com a indústria ainda é fraca; (iv) a participação internacional é baixa; e finalmente, (vi) há uma infima quantidade de trabalho escritos em inglês. 


\section{Introdução}

O avanço tecnológico e a criação de novos métodos e técnicas para desenvolvimento de software não foram as únicas iniciativas adotadas pela Tecnologia da Informação (TI) para buscar o sucesso dos projetos. A qualidade do produto que era exigida no processo de produção industrial influenciou a criação da qualidade do produto de software [Paulk 1994].

O tema Qualidade de Software tem sido objeto de ações das políticas públicas para a área de Tecnologia da Informação no Brasil. O foco é estimular a adoção de melhores práticas de engenharia de software pelas empresas brasileiras como um dos principais instrumentos para elevar a competitividade e a capacidade produtiva do setor [MCT/SEPIN 2009].

O Simpósio Brasileiro de Qualidade de Software (SBQS) é um evento anual da Comissão Especial de Engenharia de Software da Sociedade Brasileira de Computação (SBC) e do Comitê do Programa Brasileiro da Qualidade e Produtividade em Software (PBQP-SW) e é reconhecido pela SBC. O SBQS acontece desde 2002, sempre com o objetivo de reunir pesquisadores, estudantes, professores, empresários e profissionais para discutir questões que envolvem a Qualidade de Software. É válido lembrar que o simpósio promove além da troca de experiências, uma maior integração entre academia e indústria.

Em 2013, o SBQS realiza sua décima segunda edição, e este artigo tem como objetivo apresentar uma análise dos trabalhos técnicos e relatos de experiências, publicados nas últimas cinco edições do simpósio. Não se considerou os anos anteriores a 2008 devido a dificuldade de obter as informações disponíveis online, uma vez que não foi possível encontrar os sites das edições anteriores, com os trabalhos aceitos.

Este trabalho está organizado da seguinte maneira: a seção 2 apresenta os trabalhos relacionados; a seção 3 aborda o critério de análise; a seção 4 apresenta a metodologia utilizada neste trabalho; na seção 5 é feita a análise dos resultados, e finalmente, a seção 6 apresenta as considerações finais e finalmente, a seção 7 apresenta perspectivas de trabalhos futuros.

\section{Trabalhos Relacionados}

Existem poucos estudos analisando a pesquisa em Qualidade de Software no Brasil. Grande parte do esforço é realizado pelo Ministério de Ciência e Tecnologia. Porém, o foco principal das pesquisas do Ministério são voltados para a qualidade no setor de software na indústria.

Nascimento (2001) analisou a evolução da qualidade no setor de software brasileiro. O autor apresenta uma visão geral do projeto de pesquisa concebido em 1993 e é conduzido pelo governo brasileiro em parceria com diferentes agentes responsáveis pela formulação e execução da Política de Software no país.

Em 2006, Weber et al. apresentaram uma visão geral do PBQP Software, através do resumo dos resultados dos ciclos anuais dos projetos do PBQP. Os autores fizeram uma análise dos 13 anos de existência do projeto ressaltando a forte interação entre representantes do governo, academia e indústria. 
Santos (2011) apresentou um estudo baseado em revisão sistemática da literatura que procurou avaliar a influência e o impacto do MPS.BR na produção científica relacionada a Qualidade de Software no país.

Além disso, Leite et al. (2011) analisaram cinco edições do Simpósio Brasileiro de Engenharia de Software (SBES) e Silveira Neto et al. (2013) analisaram 25 edições do SBES além de realizar uma pesquisa com especialistas em Engenharia de Software.

Apesar dos estudos realizados até o momento, poucos deles levam em consideração a influência que o SBQS exerce sobre a comunidade, seja ela acadêmica ou profissional. Existe uma necessidade de analisar o impacto que o simpósio vem exercendo sobre o incentivo a Qualidade de Software no Brasil.

\section{Critério de Análise}

Esse estudo tem como objetivo fornecer uma visão geral dos artigos publicados no SBQS e sua relevância.

\subsection{Perfil dos Pesquisadores do SBQS}

A análise do perfil dos pesquisadores foi realizada considerando quem são os autores, sua filiação e estado.

\subsection{Temas de Interesse}

As publicações do SBQS são organizadas de acordo com temas de interesse. Analisouse as palavras chave, título e resumo de cada trabalho. Para classificar os temas nós utilizamos os tópicos de interesse do simpósio, que são os seguintes:

- Qualidade do Processo e/ou Produto de Software;

- Avaliação de Processo e/ou Produto de Software;

- Melhoria do Processo e/ou Produto de Software;

- Modelagem de Processo de Software;

- Modelos e Normas na Área de Qualidade de Software;

- Educação em Qualidade de Software;

- Qualidade de Software na Web;

- Qualidade de Software Orientado a Objeto;

- Qualidade e Métodos Ágeis;

- Qualidade de Software e Reutilização;

- Qualidade de Software e Gestão de Conhecimento;

- Qualidade de Software e Manutenção;

- Ferramentas para Qualidade de Software;

- Gerência e/ ou Garantia de Qualidade de Software; e,

- Verificação, Validação e Teste. 


\section{Método de Pesquisa}

Analisou-se 154 artigos (100 Trabalhos Técnicos e 54 Relatos de Experiência) do SBQS publicados entre 2008 e 2012.

\subsection{Processo de Busca}

Inicialmente os websites de cada edição do evento foram investigados, em busca dos trabalhos aceitos. Depois disso, foram analisados os anais de cada ano. O primeiro passo foi identificar informações básicas como título, autores, filiação, resumo e referencias. Estas informações foram utilizadas para fornecer uma visão geral dos trabalhos.

As publicações do simpósio são classificadas em Trabalhos Técnicos (TT) e Relatos de Experiência (RE). Para os trabalhos técnicos são selecionados artigos descrevendo resultados inéditos sobre pesquisas na área de Qualidade de Software com contribuição acadêmica. Já nos relatos de experiência são selecionados artigos descrevendo e analisando a aplicação de processos, métodos ou ferramentas de Qualidade de Software, contextualizando a experiência e mostrando os resultados obtidos e lições aprendidas, em uma experiência prática com contribuição para a indústria de software.

A Figura 1 apresenta a quantidade de trabalhos técnicos e relatos de experiência ao longo das últimas 5 edições do SBQS. É possível notar um aumento na quantidade de RE no ano de 2010, porém nos anos seguintes este número cai, atingindo a menor quantidade de publicações no ano de 2012.

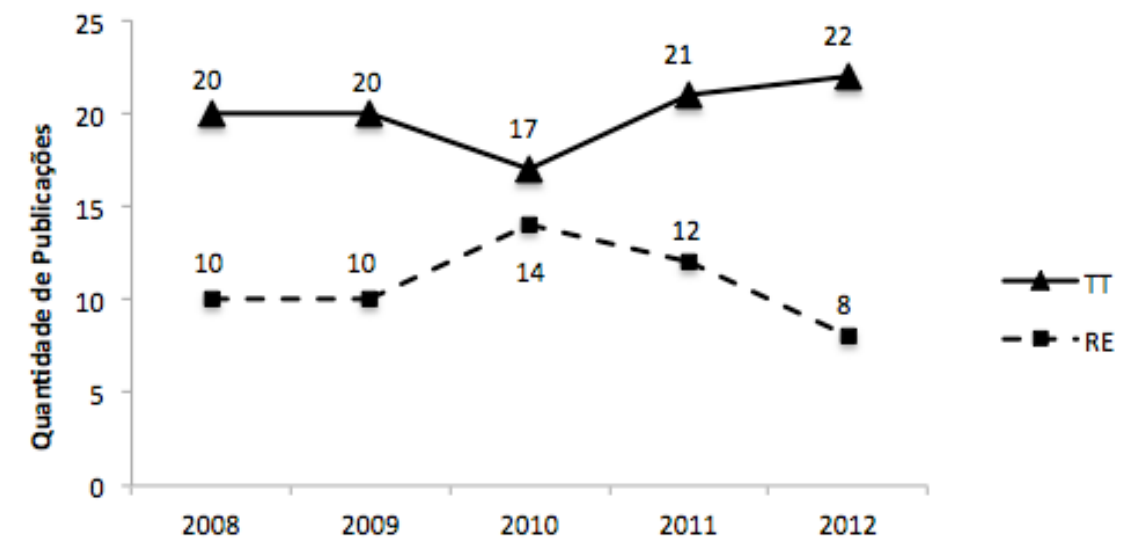

Figura 1 - Quantidade de Trabalhos Técnicos vs. Relatos de Experiência

\subsection{Questões de Pesquisa}

Com o intuito de obter uma visão geral das publicações do SBQS, considerando as últimas cinco edições do evento e identificar o perfil dos pesquisadores, foram definidas as seguintes questões de pesquisa:

1. Quais são as instituições com o maior número de publicações?

2. Quais são os autores mais frequentes em publicações do SBQS?

Para identificar qual o tipo de pesquisa relacionado a Qualidade de Software tem maior foco no SBQS, as seguintes questões de pesquisa foram definidas: 
3. Quais são os tópicos de pesquisa mais investigados?

Para analisar o relacionamento das publicações com a indústria e como as artigos do SBQS são referenciados, definiu-se as seguintes questões de Pesquisa:

4. Quais são as instituições que mais contribuem para aproximação da academia com a indústria?

5. Quais os artigos das versões anteriores do SBQS que foram mais referenciados nos últimos cinco anos?

\section{Análise dos Resultados}

A Figura 2 apresenta a participação dos estados. O grande número de trabalhos vindos de instituições da região sul/sudeste (RJ, MG, SP, SC, entre outras) pode ser justificado pelo grande número de organizações (empresas de desenvolvimento de software em sua maioria) que está concentrado nesta região $(77,8 \%)$ [MCT/SEPIN 2009].

O estado de Pernambuco possui um diferencial em relação aos demais nordestinos. Ele abriga em sua capital (Recife) o maior parque tecnológico da região, o Porto Digital. Com incentivos do Governo do Estado de Pernambuco e investimento de empresas privadas do setor de Tecnologia da Informação (TI). Além disso, as universidades locais atuam fortemente desenvolvendo projetos e parcerias com as empresas do parque.

Hoje, o Porto Digital abriga centenas de empresas do ramo tecnológico. Cerca de $74 \%$ destas estão voltadas para o desenvolvimento de software. Grandes empresas como Microsoft, IBM e Samsung possuem bases instaladas no parque.

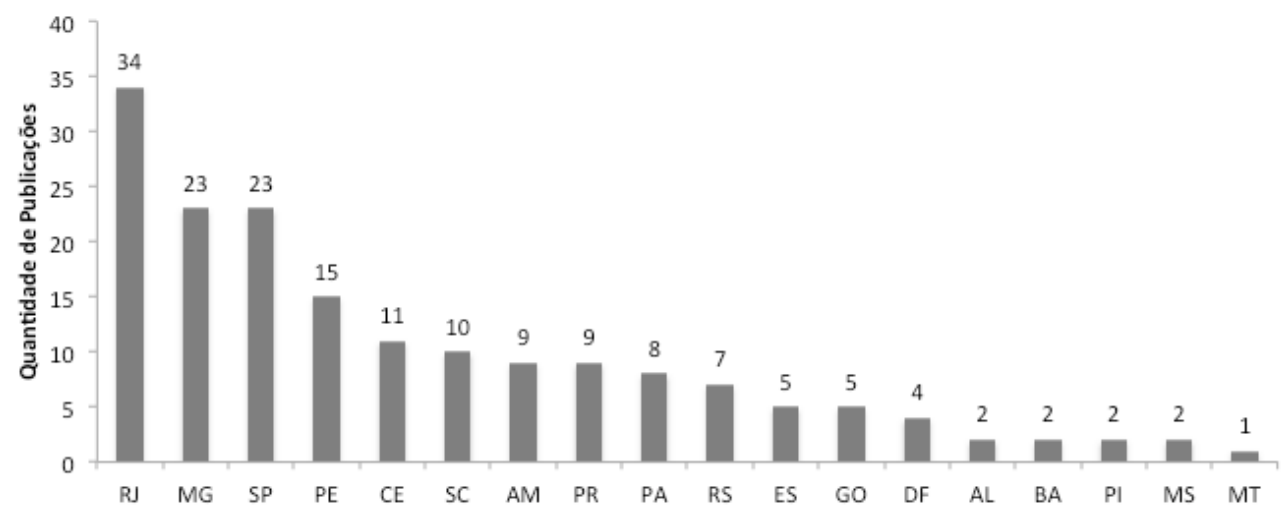

Figura 2 - Participação por Estado

A Figura 3 apresenta a quantidade de publicações das Instituições ao longo dos 5 anos de realização do evento. 


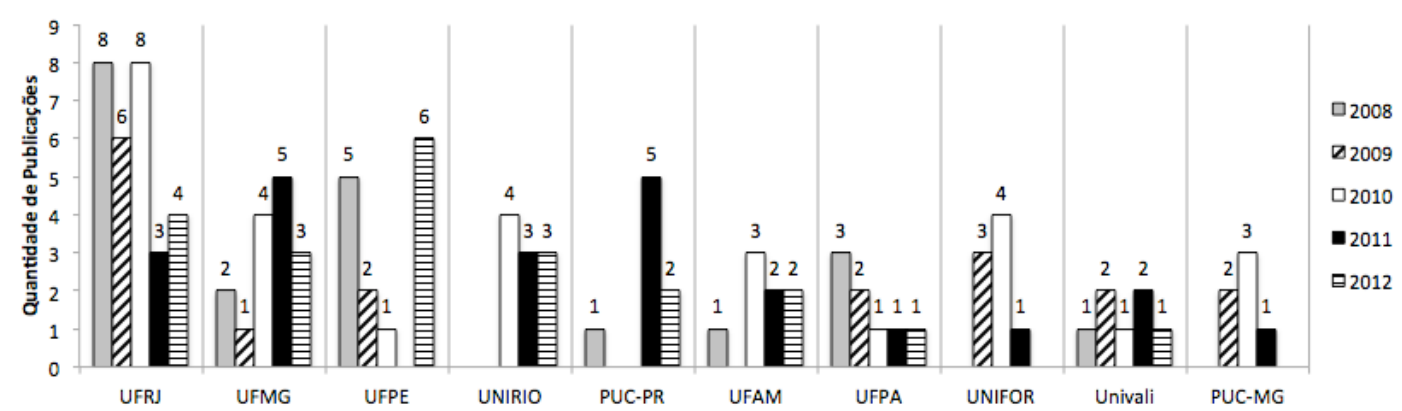

Figura 3 - Participação das Instituições nos Últimos 5 anos

A UFRJ embora seja a mais produtiva, apresentou uma queda significativa nos últimos dois anos do simpósio. Este declínio pode ser justificado pela mudança de vínculo do pesquisador Gleison Santos da UFRJ para UNIRIO. O que consequentemente comprova o aumento da participação da UNIRIO a partir de 2010. Além disso, foi possível identificar um aumento da participação de outras instituições como a UFAM, PUC-PR, UFMG, entre outras.

Outro fato que pode ter interferido na redução da participação da UFRJ é que em 2009 a Petrobrás anunciou o investimento de US\$ 10 milhões na construção de um centro internacional de pesquisa para a área do pré-sal. Este centro que ainda será construído ficará no Parque Tecnológico da Universidade Federal do Rio de Janeiro, diante dessa informação acredita-se que o foco de pesquisa tenha mudado.

A segunda instituição que mais contribuiu com publicações de TT e RE foi a UFMG. A universidade mantém participação frequente ao longo dos últimos cinco anos, demonstrando um aumento considerável a partir de 2010.

A UFPE também participa ativamente no simpósio. Percebemos que o número de publicações da universidade vinha caindo chegando ao ponto de não ter nenhum trabalho aceito no ano de 2011. A esta ocorrência pode-se atribuir o fato do professor pesquisador Alexandre Vasconcelos ter se ausentado da instituição nos anos de 2010 e 2011 para iniciar seu pós-doutorado na Espanha.

Além disso, podemos destacar a participação da UNIVALI, UFPA e UFAM. Apesar de tímida, as três instituições vêem mantendo a representação de forma constante ao longo dos últimos cinco anos. A UNIRIO passou a ter uma contribuição significativa a partir de 2010.

O norte do Brasil está sendo representado pela UFAM e UFPA. Juntas elas somam 16 publicações. UFPA deve esta constante participação ao Laboratório de Engenharia de Software (Labes) fundado em 2004 no campus da instituição. O Labes atualmente encontra-se entre os 10 melhores grupos de pesquisa na área de processo de software da América Latina

A Figura 4 apresenta a classificação dos 10 autores que mais publicaram nas últimas 5 edições do SBQS. 368 pesquisadores participaram em pelo menos um dos 154 trabalhos, sendo que a maioria é autor de apenas um artigo. Os autores mais produtivos possuem vínculo com a UFRJ. A Ana Regina Rocha e o Gleison Santos são os únicos que publicaram em todos os cinco anos analisados. O segundo grupo mais produtivo é da PUC-PR, sendo representada por Sheila Reinehr e Andreia Malucelli. Por fim, Marco Valente representa a UFMG e Alexandre Vasconcelos a UFPE. 


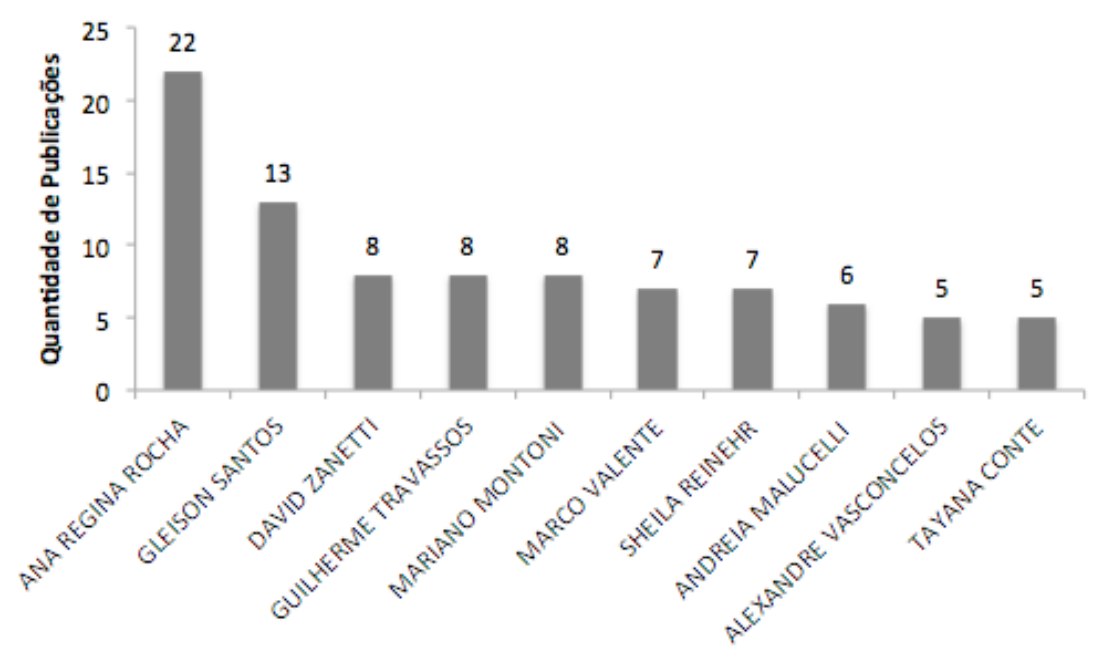

Figura 4 - Top 10 dos autores que mais publicaram no SBQS

A Tabela 1 apresenta quais são os 5 temas de interesses mais pesquisados no SBQS. Durante o registro dos artigos os autores podem classificar a publicação em mais de uma categoria, porém definimos apenas uma categoria por publicação, geralmente aquela que abrange a maior área de contribuição.

A grande maioria dos trabalhos focam na melhoria do processo e/ou produto de software (38), boa parte deste número pode ser justificado pelas contribuições geradas pela parceria entre COPPE e UFRJ. Outro tópico bastante explorado foi o de verificação, validação e teste. Este último abrange diversos subtópicos como inspeção, testes unitários, integração, usabilidade, exploratório, caixa preta, entre outros.

O número de artigos descrevendo Ferramentas para Qualidade de Software caiu ao longo dos últimos 5 anos, o que pode justificar a queda na quantidade de artigos de RE. Além disso, nós identificamos que o número de artigos descrevendo Qualidade e Métodos ágeis apesar de serem poucos, permanecem frequentes ao longo dos anos.

Tabela 1. Temas de Interesse

\begin{tabular}{|l|c|c|c|c|c|c|}
\hline \multicolumn{1}{|c|}{ Tópico } & $\mathbf{2 0 0 8}$ & $\mathbf{2 0 0 9}$ & $\mathbf{2 0 1 0}$ & $\mathbf{2 0 1 1}$ & $\mathbf{2 0 1 2}$ & Total \\
\hline Melhoria do Processo e/ou Produto de Software & 6 & 9 & 6 & 9 & 8 & 38 \\
\hline Verificação, Validação e Teste & 7 & 2 & 8 & 3 & 8 & 28 \\
\hline Avaliação de Processo e/ou Produto de Software & 4 & 4 & 3 & 1 & 2 & 14 \\
\hline Qualidade e Métodos Ágeis & 2 & 2 & 3 & 2 & 3 & 12 \\
\hline Ferramentas para Qualidade de Software & 4 & 3 & 3 & 1 & 0 & 11 \\
\hline Outras & 7 & 10 & 10 & 17 & 9 & 51 \\
\hline
\end{tabular}

A Tabela 2 apresenta a participação de empresas e organizações da indústria. O baixo número de publicações vindo da indústria pode ser reflexo da falta de qualificação, atualização e capacitação profissional do capital humano dentro da organização [MCT/SEPIN 2009]. 
Tabela 2. Participação da Indústria

\begin{tabular}{|l|c|}
\hline \multicolumn{1}{|c|}{ Empresa/Organização } & Qtd. \\
\hline Instituto Alberto Luiz Coimbra de Pós-Graduação e Pesquisa de Engenharia - COPPE & 28 \\
\hline Serviço Federal de Processamento de Dados - Serpro & 5 \\
\hline Instituto Atlântico & 4 \\
\hline Centro de Tecnologia da Informação Renato Archer - CTI & 3 \\
\hline Instituto Nokia de Tecnologia - INdT & 3 \\
\hline Associação Sul-Riograndense de Apoio ao Desenvolvimento de Software - SOFTSUL & 2 \\
\hline Centro de Estudos e Sistemas Avançados do Recife - CESAR & 2 \\
\hline Fundação Centro de Análise, Pesquisa e Inovação Tecnológica - Fucapi & 2 \\
\hline IBM Research & 1 \\
\hline Secretaria Executiva da Fazenda do Estado de Alagoas - SEFAZ-AL & 1 \\
\hline Outras & 51 \\
\hline
\end{tabular}

Esse cenário deveria ser diferente, uma vez que em 2007 foi sancionada a Lei ${ }^{\circ}$ 11.487, de incentivo à ciência e tecnologia. Ela permite a redução fiscal das empresas que investirem em projetos desenvolvidos por instituições públicas. O objetivo dessa medida é aproximar o setor produtivo e industrial da pesquisa acadêmica aplicada.

A COPPE foi a instituição que mais contribuiu para que a aproximação da academia com a indústria se tornasse realidade. Em 1994, o instituto criou a Incubadora por onde já passaram diversas empresas. Além disso, a COPPE também é uma instituição implementadora e avaliadora do MPS.BR credenciada pela SOFTEX. Ela tem atuado na implementação de melhoria de processos de software em micro, pequenas, médias e grandes empresas do país.

O Serviço Federal de Processamento de Dados (Serpro) é uma empresa pública, vinculada ao Ministério da Fazenda, cujo negócio é a prestação de serviços em Tecnologia da Informação e Comunicações para o setor público. Ela é considerada uma das maiores organizações do setor, na América Latina. O Serpro aposta em parcerias com instituições públicas de ensino superior espalhadas pelo país para desenvolver pesquisas, focadas em governo eletrônico.

Em geral são convênios para transferência de recursos, mas há também acordos que não envolvem repasses, como no caso da parceria com a UFBA na refatoração de código para uso do framework Demoiselle. A parceria com a UFSC possibilitou dois importantes convênios até o momento. $\mathrm{O}$ primeiro já encerrado, garantiu a cooperação técnico-científica para execução de engenharia reversa e aperfeiçoamento da versão online do Sistema de Gerenciamento e Certificados do Serpro. O segundo, em fase final de formulação, prevê a adequação do software da Autoridade Certificadora Serpro (ACSerpro) aos requisitos estabelecidos pela infraestrutura de Chaves Públicas Brasileiras (ICP-Brasil). Outro convênio em andamento é com a UFPR para pesquisa tecnológica sobre persistência de dados em ambientes baseados em software livre.

Outra empresa que merece destaque é o Instituto Atlântico. Ela foi a primeira organização do Norte e Nordeste a conquistar o CMMI Nível 3 (Capability Maturity Model Integration). Esta qualificação abrange todos os processos da área de desenvolvimento de software, atesta a auditoria da ISD Brasil. Sua sede, localizada em Fortaleza disponibiliza ampla infra-estrutura para suporte à pesquisa e desenvolvimento de soluções tecnológicas, contando com áreas específicas para laboratórios de desenvolvimento de aplicações de software e hardware, laboratórios de teste, sala de treinamento, auditórios e espaço de convivência. Possui três filiais, Sobral e Tauá ambas interior do Ceará e outra em São Paulo, sendo que esta ultima firmou parceria com a 
empresa de desenvolvimento Altec e ambas concentram seus esforços no desenvolvimento de aplicações e na realização de testes de software.

Apesar de uma grande participação das empresas no simpósio, é possível perceber que as publicações vindas de empresas não são constantes. O que pode ser justificado pelo fato da publicação em si não trazer retorno financeiro para as empresas. Outro fator que pode afetar a participação da indústria é o nível de confidencialidade dos projetos desenvolvidos. Afim de proteger suas estratégias e novas técnicas, algumas empresas evitam a publicação dos seus relatos de experiência.

A Figura 5 apresenta o relacionamento entre a quantidade de publicações e a língua utilizada na escrita dos artigos. No ano de 2008, duas publicações foram de instituições de Portugal e uma da Nova Zelândia. Já em 2012, as duas publicações em espanhol foram realizadas por instituições peruanas. Podemos destacar também neste ano, duas publicações de instituições portuguesas. A grande maioria dos trabalhos escritos em inglês são de instituições brasileiras.

Finalmente, apesar do aumento da quantidade de publicações em línguas estrangeiras, o número de artigos em português ainda é um empecilho para obtenção de uma melhor qualificação junto a CAPES ${ }^{1}$. Uma prática utilizada por alguns eventos (e.g. SBES) para aumentar a possibilidade de participação internacional é submissão da chamada para participação em inglês e a tradução do web site do evento.

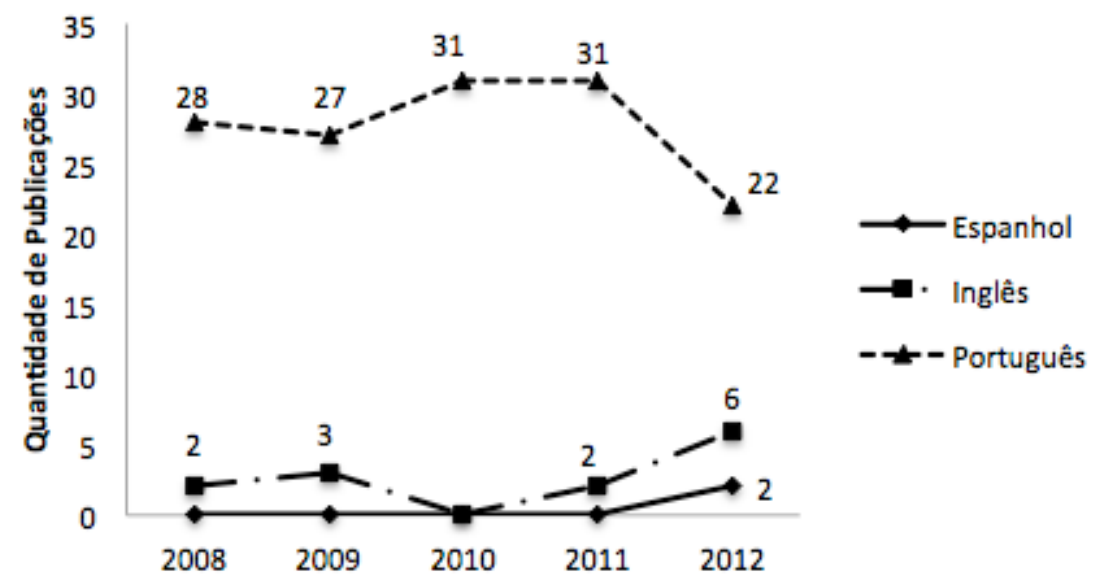

Figura 5 - Línguas usadas na escrita dos artigos

Para análise da quantidade de referencias a artigos do SBQS, foram analisados todas as referencias dos artigos publicados entre os anos 2008 e 2012 através de uma técnica conhecida como "snow-balling" [Runeson 2009].

A figura 6 apresenta a quantidade de referências a artigos do SBQS ao longo dos últimos 5 anos do simpósio. Ao todo, 112 trabalhos das edições foram citados, sendo que 2011 foi o ano com o maior número de citações (30) com uma média de 1,1 referências por artigos.

\footnotetext{
${ }^{1}$ http://www.capes.gov.br/avaliacao/qualis
} 
Particularmente, estes números foram alcançados por causa de 2 artigos, 1 relatos de experiência [Bettio et al. 2011] (referenciando 9 trabalhos) e 1 trabalho técnico [Carvalho et al. 2011] (referenciando 5 trabalhos).

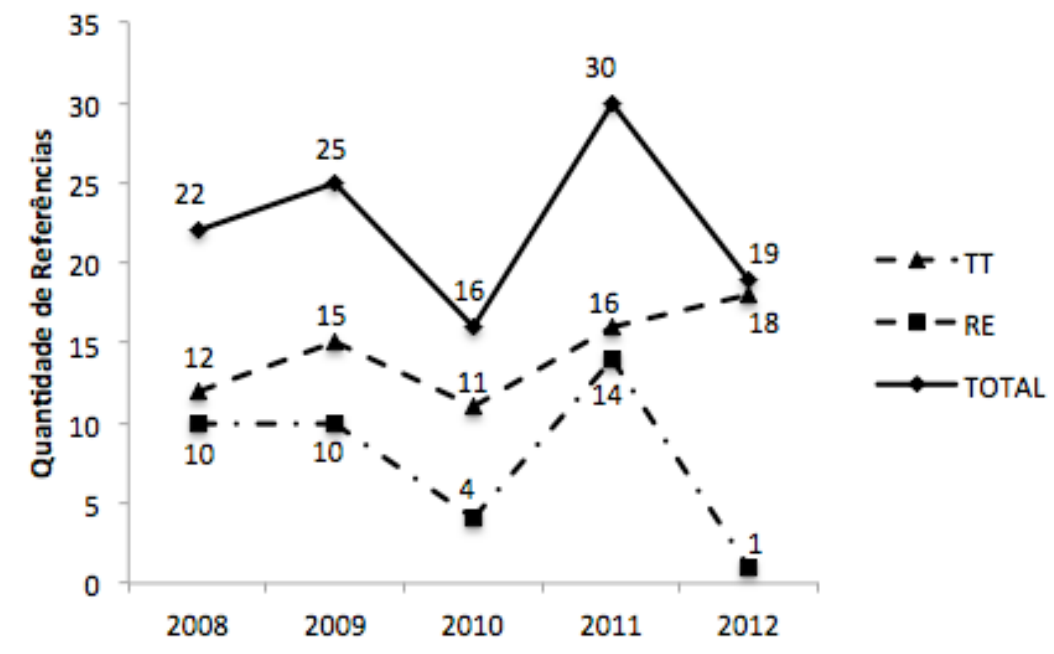

Figura 6 - Quantidade de referencias ao longo dos últimos 5 anos

Apesar da queda na quantidade de referências em 2012, média de artigos referenciados nos Trabalhos Técnicos ao longo dos cinco anos ficou em torno de 14 referências por ano e desde 2010 apresentam um bom crescimento. Ao contrário do número de artigos referenciados nos Relatos de Experiência, cuja média ficou em torno de 7 referências por ano. Provavelmente esta instabilidade na quantidade de trabalhos referenciados nos RE se deva a dois fatores. O primeiro está relacionado a participação de empresas e profissionais que nunca tiveram contato prévio com os trabalhos do simpósio. Já o segundo fator pode estar relacionado com as experiências que nunca foram exploradas anteriormente.

Finalmente, os trabalhos do ano de 2006 foram os mais referenciados (32 citações). Os artigos mais citado pelos autores foram de Montoni et al. (2006) e Santos et al. (2009). Sendo cada um deles citados 4 vezes em diferentes artigos ao longo dos cinco anos de SBQS.

\section{Conclusão}

Nossa análise considerou a versão eletrônica das últimas cinco edições do Simpósio Brasileiro de Qualidade de Software através da investigação dos 154 artigos publicados nas seções de trabalhos técnicos e relatos de experiência do evento.

As informações levantadas nos permitiram chegar às seguintes conclusões: (i) as instituições que lideram o ranking de publicações do SBQS são da região Sul e Sudeste, a liderança é da UFRJ que sozinha foi responsável por $19 \%$ das publicações do simpósio, seguida da UFMG (10\%) e UFPE (9\%); (ii) os autores mais frequentes são Ana Regina Rocha (14\%), Gleison Santos (8\%), David Zanetti (5\%), Guilherme Travassos (5\%) e Mariano Montoni (5\%); (iii) os tópicos de pesquisa mais investigados são focados na Melhoria do Processo e/ou Produto de Software (25\%), Verificação, Validação e Teste (18\%) e Avaliação de Processo e/ou Produto de Software (9\%); (iv) as instituições que mais contribuíram para aproximação entre academia e indústria 
foram a parceria entre COPPE e UFRJ (18\%); e finalmente (v) os artigos mais referenciados nos artigos da SBQS foram [Montoni et al. 2006] e [Santos 2009].

Sem dúvidas o SBQS desempenha um papel fundamental no desenvolvimento da Qualidade de Software no país, além de facilitar a comunicação entre academia e indústria. Porém, ainda existe muito trabalho a ser feito [MCT/SEPIN 2009].

Como forma de estímulo a participação da indústria, o simpósio poderia promover um concurso aonde o ganhador receberia um prêmio remunerado. $\mathrm{O}$ que justificaria o compartilhamento de relatos de experiência.

A quantidade de referencias a artigos do SBQS ainda é muito baixa. A média geral de citações ao longo dos cinco anos de eventos é de 0,7 referencias por artigo. Provavelmente a dificuldade de encontrar as publicações anteriores é um dos grandes responsáveis por esta média.

Finalmente, apesar de existir uma boa colaboração entre as Universidades, e algumas parcerias isoladas entre empresas e instituições, a relação com a indústria ainda precisa ser fortalecida. Além disso, a participação internacional é quase nula e também precisa ser estimulada.

\section{Trabalhos Futuros}

Como proposta de trabalhos futuros, pretende-se estender a pesquisa para todos os anos anteriores do SQBS. Para isto, precisa-se ter acesso a todas as versões dos anais anteriores. Desta forma, propõe-se a criação de um web site que permita a comercialização ou disponibilização dos anais de todas as edições anteriores.

Além disso, pretende-se também incluir a análise do Concurso de Teses e Dissertações em Qualidade de Software (CTDQS) que é realizado em conjunto com o SBQS. O CTDQS visa divulgar e premiar trabalhos de tese de doutorado e de dissertações de mestrado na área de qualidade. Acredita-se que os trabalhos desenvolvidos no concurso são os pioneiros com relação a pesquisa sobre Qualidade no país.

\section{Referências}

Bettio, K., Valaski, J., Gomes, D., Matias, E., Reinehr, S., Malucelli, “A. Uma Experiência de Implementação Nível G em uma Empresa de Software Livre”, Em: X Simpósio Brasileiro de Qualidade de Software - SBQS 2011, Paraná - PR, Junho 2011.

Carvalho, D., Costa, A., Sales, E., Lima, A., Reis, R. “Apoio à Reutilização de Processos de Software em um Ambiente de Engenharia de Software Centrado em Processo". Em X Simpósio Brasileiro de Qualidade de Software - SBQS 2011, Paraná - PR, Junho 2011.

Leite, J., Batista, T. e Leite, L. (2011) "Software Engineering Research in Brazil: An Analysis of the Last Five Editions of SBES", Em: XXV Simpósio Brasileiro em Engenharia de Software, pág. 24 a 29.

MCT/SEPIN (2009) "Pesquisa de Qualidade no Setor de Software Brasileiro", Em: Ministério da Ciência e Tecnologia / Secretaria de Política de Informática. Brasília, Brasil. 
Montoni, M., Santos, G., Figueiredo, S., Cabral, R., Barcelos, R., Barreto, A., Barreto, A., Cerdeiral, C., Lupo, P., Rocha, A. R. (2006) "Uma Abordagem de Garantia de Qualidade de Processos e Produtos de Software com Apoio de Gerência de Conhecimento na Estação TABA". Em: V Simpósio Brasileiro de Qualidade de Software - SBQS 2006 - Vila Velha, Brasil, 2006.

Nascimento, C. J. (2001) “A Evolução da Qualidade no Setor de Software Brasileiro: Quatro Biênios medindo e Acompanhando Indicadores de Gestão”, Em: IV Encontro para a Qualidade nas Tecnologias de Informação e Comunicações. Lisboa, Portugal.

Paulk, M., (1994) "A Comparison of ISO 9001 and the Capability Maturity Model for Software", Software Engineering Institute, Carnegie Mellon University, Pittsburgh, Pennsylvania, Technical Report CMU/SEI-94-TR-012.

Runeson, P., Skoglund, M., "Reference-based search strategies in systematic reviews", Em: 13th International Conference on Empirical Assessment \& Evaluation in Software Engineering, Durham University, UK, 2009.

Santos, G., Katsurayama, A.E., Montoni, M., et al. (2009), "Lições Aprendidas em uma Iniciativa de Melhoria de Processos de Software sob Diferentes Perspectivas: Membros da Organização, Implementadores e Avaliadores". Em: VIII Simpósio Brasileiro de Qualidade de Software - SBQS 2009, Ouro Preto - MG, Junho 2009.

Santos, G. (2011), "Influência e Impacto do Programa MPS.BR na Pesquisa Relacionada à Qualidade de Software no Brasil”. Em: X Simpósio Brasileiro de Qualidade de Software - SBQS 2011, Paraná - PR, Junho 2011.

Silveira Neto, P. A. M., Gomes, J. S., Almeida, E. S., Leite, J. C., Batista, T. V., Leite, L. "25 Years of Software Engineering in Brazil: Beyond an insider's view", Em: Journal of System and Software, pág. 872 a 889, volume 84, número 4, 2013.

Weber, K. C., Nascimento C. J., Marinho, D. S. (2006) "Programa Brasileiro da Qualidade e Produtividade em Software: Treze anos acompanhando e disseminando a cultura da qualidade", Em: Revista ProQualiti - Qualidade na Produção de Software, vol. 2, num. 1. Lavras, Brasil. 